\section{REVIEWING RECORDINGS OF NEONATAL RESUSCITATION WITH PARENTS}

Few of us relish the thought of our performance in a challenging situation being recorded and reviewed by others, but many have accepted it for research purposes in the context of newborn resuscitation. At Leiden University Medical Centre Neonatal Unit they have been recording videos of all newborn resuscitations since 2014 in order to study and improve care during transition. The recordings are kept as a part of the medical record and, in contrast with other published practice to date, parents are offered an opportunity to review the recording with a professional and to have still images from it or a copy of the video. In this qualitative study Maria C den Boer and colleagues interviewed parents of preterm babies who had viewed their baby's recording to provide insight into their experience. The study included 25 parents of 31 preterm babies with median gestational age $27+5$ weeks. Four of the babies had gone on to die in the neonatal unit. Most parents offered the opportunity to see the recording wished to do so and around two thirds asked for images or a copy. The parental experiences of viewing the videos were very positive. The experience improved their understanding of what had happened, enhanced their family relationships, and increased their appreciation of the care team.

Colm O'Donnell discusses his own experience with researching video recordings of resuscitation, beginning with a visit to Neil Finer and Wade Rich at University of California, San Diego in 2003. Colm also has positive experiences of sharing the recordings with families. The team in Leiden recommend this practice. Both articles are an interesting read that will challenge your assumptions and stimulate reflection. See page F346 and F344

\section{PHYSIOLOGICAL RESPONSES TO FACEMASK APPLICATION IN NEWBORNS IMMEDIATELY AFTER BIRTH}

Vincent Gaertner and colleagues reviewed video recordings of initial stabilisation at birth of term and late-preterm infants who were enrolled in a randomised trial of different face-masks. 128 face-mask applications were evaluated. In eleven percent of face-mask applications the infant stopped breathing. When apnoea occurred after mask application there was a median fall in heart rate of 38 beats per minute. These episodes are considered to represent the trigeminocardiac reflex and recovered within 30 s. Apnoea was also observed after face-mask reapplications, although less frequently. There were a median of 4 facemask applications per infant, suggesting a lot of additional potential for avoidable interruption of support. This observation of apneoa after face-mask application is less frequent than in previous reports in more preterm infants but is still quite common. See page F381

\section{OUTCOMES OF A UNIFORMLY ACTIVE APPROACH TO INFANTS BORN AT 22- 24 WEEKS OF GESTATION}

This single centre report by Fanny Söderström and colleagues from Uppsala in Sweden describes the outcomes of infants born at 22 to 24 weeks gestation between 2006 and 2015. In this institution, all mother-infant dyads at risk for extremely preterm delivery are provided proactive treatment. This includes intrauterine referral when approaching 22 weeks of gestation, provision of tocolytics, antenatal steroids and family counselling. There were 222 liveborn infants born at the hospital or admitted soon after birth. There had been four fetal deaths during in utero transport to the centre and there were 14 stillbirths of fetuses that were alive at admission. Two infants died in the delivery room after birth. Survival of the liveborn babies was $52 \%$ at 22 weeks, $64 \%$ at 23 weeks and $70 \%$ at 25 weeks. Follow-up information was available for $93 \%$ of infants. There were 10 infants with cerebral palsy and no infants who were blind or deaf. Around a third had diagnosis of developmental delay. The study provides a measure of what can be achieved when decisions to initiate treatment are not selective according to the views of the parents and physicians. See page F413

\section{BRONCHOPULMONARY DYSPLASIA AND GROWTH}

Theodore Dassios and colleagues analysed data from the UK National Neonatal Research Database for the years 2014 to 2018. They looked at postnatal growth in all liveborn infants born before 28 weeks gestation and admitted to neonatal units. There were 11806 infants. Bronchopulmonary dysplsia was defined as any requirement for respiratory support at 36 weeks and affected $57 \%$. As measured by change in weight and head circumference z-scores from birth to discharge, the infants who developed BPD grew slightly better than those who did not. See page F386

\section{DISORDERS OF VISION IN NEONATAL HYPOXIC-ISCHAEMIC ENCEPHALOPATHY}

Eva Nagy and colleagues undertook a systematic review of reports of outcome after hypoxic ischaemic encephalopathy to evaluate the evidence relating to visual impairment. Although this is a recognised complication of hypoxic ischaemic encephalopathy, it has not been well described. They identified six studies that enrolled 283 term born infants that met their inclusion criteria. Some form of visual impairment was reported in 35\% but there was huge variation in the techniques used for assessment. It remains difficult to advise families about the risks and nature of visual impairments that might be encountered. There are lots of barriers to obtaining good information in this area because of the need for prolonged follow-up and difficulty in testing individuals with other difficulties. See page F357

\section{MANAGEMENT OF SYSTEMIC HYPOTENSION IN TERM INFANTS WITH PERSISTENT PULMONARY HYPERTENSION OF THE NEWBORN}

Heather Siefkes and Satyan Lakshminrusimha present a beautifully illustrated review of the multiple factors contributing to haemodynamic disturbance in infants with PPHN, and the mechanisms of action of the various candidate therapeutic agents. This supports a reasoned approach to treatment. The challenge remains to supplement this with high quality evidence. The HIP trial report illustrates the enormous challenge of studying treatments for haemodynamic disturbance in the immediate newborn period and the hurdles that need to be overcome to enable progress. See page F446 and F398 